\title{
Modeling of Shock Waves in Two-Dimensional Electron Channels: Effect of Tsunami
}

\author{
I. Semenikhin*, E. Vostrikova ${ }^{\dagger}$, A. Ivanov ${ }^{\dagger}$, and V. Ryzhii ${ }^{*}$ \\ *University of Aizu, Aizu-Wakamatsu 965-9580, Japan \\ v-ryzhii@u-aizu.ac.jp \\ ${ }^{\dagger}$ Moscow Institute of Physics and Technology, Moscow 141700, Russia \\ ekaterinavost@mail.ru
}

\begin{abstract}
Formation of shock waves in two-dimensional electron channels by electrical signals is studied using analytical and numerical models based on hydrodynamic electron transport equations coupled with two-dimensional Poisson equation.
\end{abstract}

\section{Introduction}

Plasma waves in two-dimensional electron gas (2DEG) channels can be used in different heterostructure electron devices operating in the terahertz range of frequencies [1]. Theoretical and experimental studies of the plasma effect in the 2DEG channels, in particular, in the 2DEG channels with highly conducting gates, have been reported in many publications.

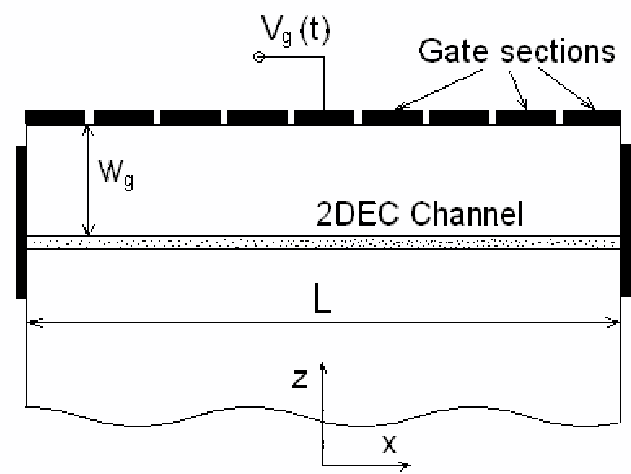

Figure 1: Schematic view of the structure with 2DEG gated channel under consideration. The side contacts correspond to $x= \pm L / 2$. The coordinates of the 2DEG channel and the gate electrodes correspond to $z=0$ and $z=W_{g}$, respectively, where $W_{g}$ is the thickness of the gate layer. 
In this paper, we present the results of analytical and numerical investigation of strongly nonlinear phenomena in the 2DEG channel with the gate split into several section as shown in Fig. 1. It is assumed that apart from a dc voltage, $V_{0}$, a transient voltage $V_{g}(t)$ is applied to the central gate, so that the spatio-temporal distribution of the gate potential is given by $V_{g}(t)=V_{0}+V_{g} \Theta\left(|x|-l_{g} / 2\right) \cdot \Theta(t) \cdot \exp \left(-t / t_{0}\right)$. Here $\Theta(x)$ is a smoothened unity-step function, $l_{g}$ is the length of the gate section, and $t_{0}$ is the characteristic time determined by the controlling electric circuit (the $R C$-time).

\section{Model}

Our model is based on a set of the hydrodynamic equations coupled with two-dimensional Poisson equation for the self-consistent electric potential:

$$
\begin{aligned}
& \frac{\partial u}{\partial t}+v u+u \frac{\partial u}{\partial x}-K \frac{\partial^{2} u}{\partial x^{2}}=\left.\frac{e}{m} \frac{\partial \varphi}{\partial x}\right|_{z=0}, \\
& \frac{\partial \Sigma}{\partial t}+\frac{\partial \Sigma u}{\partial x}=0 \\
& \frac{\partial^{2} \varphi}{\partial x^{2}}+\frac{\partial^{2} \varphi}{\partial z^{2}}=\frac{4 \pi e}{\mathfrak{x}}\left(\Sigma-\Sigma_{d}\right) \cdot \delta(z) .
\end{aligned}
$$

Here, $u=u(t, x)$ and $\Sigma=\Sigma(t, x)$ are the electron hydrodynamic velocity along and sheet density in the 2DEG channel, $\varphi=\varphi(t, x, z)$ is the electric potential, $\Sigma_{d}$ is the donor sheet concentration in the 2DEG channel, $v$ is the frequency of the electron scattering on impurities and phonons, $K$ is the coefficient of viscosity associated with the electronelectron scattering processes, $e=|e|$ and $m$ are the electron charge and effective mass, respectively, $\mathfrak{x}$ is the dielectric constant, and $\delta(z)$ is the Dirac delta function.

The transient phenomena in the 2DEG system under consideration were analyzed both analytically and numerically. The Galerkin spectral method [2] was used in numerical calculations. Using the developed model, we demonstrated the formation and transformation of strongly nonlinear plasma waves and calculated the output transient electrical currents through different gate sections.

\section{Results}

Examples of the simulation results for uniformly doped 2DEG channels are shown in Figs. 2 and 3. The initial spatial distributions of the sectioned gate provide rather sharp initial distributions of the electron density (see, the curves corresponding to $\tau=t v=0$. As shown, at sufficiently large transient signal amplitude $V_{g}$, the formation of nonlinear waves propagating along the channel is possible followed by the formation of the shock waves. The latter occurs in the structures with relatively thin gate layers, in which the dispersion relation for the plasma waves is close to linear. The pattern of the electron density evolution is substantially different in the case of relatively large $w_{g}$ (see the electron density distributions in Fig. 3 for $w_{g}=1$ ). The latter case corresponds to "deep water" situation. 

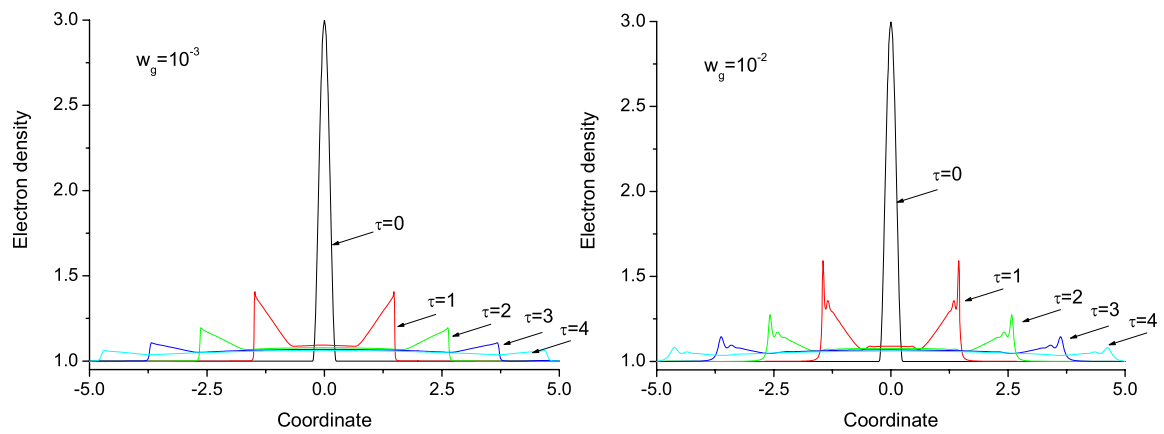

Figure 2: Spatial distributions of electron density in the structures with different normalized gate length $\mathscr{L}_{g}=L_{g} v / s \simeq L v / s=10$ and normalized gate layer thicknesses $w_{g}=W_{g} v / s=10^{-3}$ and $10^{-2}$ at different moments $\tau=t v$, where $s$ is the phase velocity of linear plasma waves in gated channel (at normalized circuit characteristic time $\tau_{0}=t_{0} v=10^{-2}$, relative electron viscosity $k=K v / s^{2}=2 \times 10^{-3}$, and $v_{g}=e V_{g} / m s^{2}=2$ ). This case is analogous to the propagation of surface waves in a "shallow water" channel.
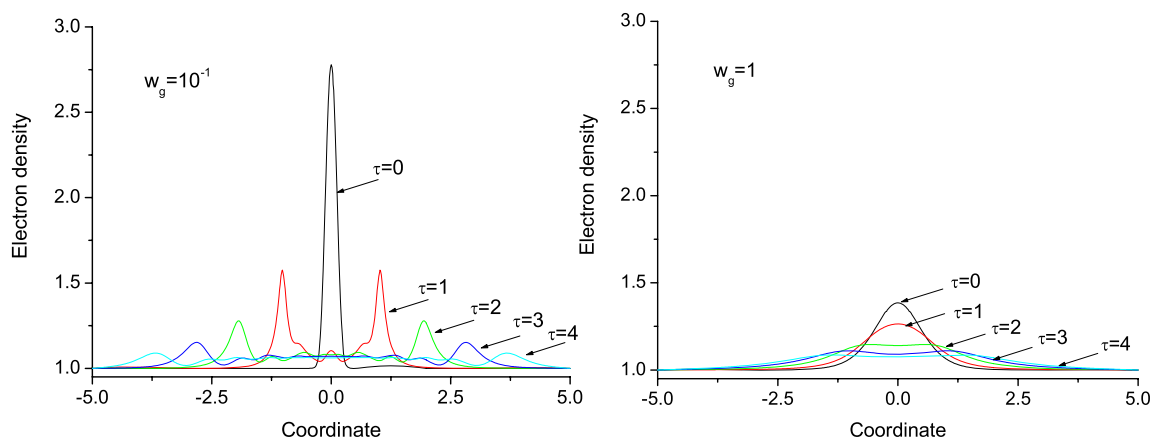

Figure 3: The same as in Fig. 2 but for $w_{g}=10^{-1}$ and $w_{g}=1$.

Figure 4 demonstrates the evolution of the normalized sheet charge $\left(\Sigma-\Sigma_{d}\right) /\left\langle\Sigma_{d}\right\rangle$ in the 2DEG channel with a nonuniform donor distribution exshibiting a maximum in the channel center. Here $\left\langle\Sigma_{d}\right\rangle$ is the average donor density. One can see that the wave fronts in the channel central part (where the donor density is large) are relatively smooth, but when the approaches the side areas with small donor density, the fronts become rather sharp. The formation of the secondary fronts is also seen. This situation is akin to the formation of the waves on the surface of the ocean with a decreasing depth near the cost (smaller donor density corresponds to smaller depth of water). The simulated phenomena resemble those associated with the tsunami effect in the ocean. 


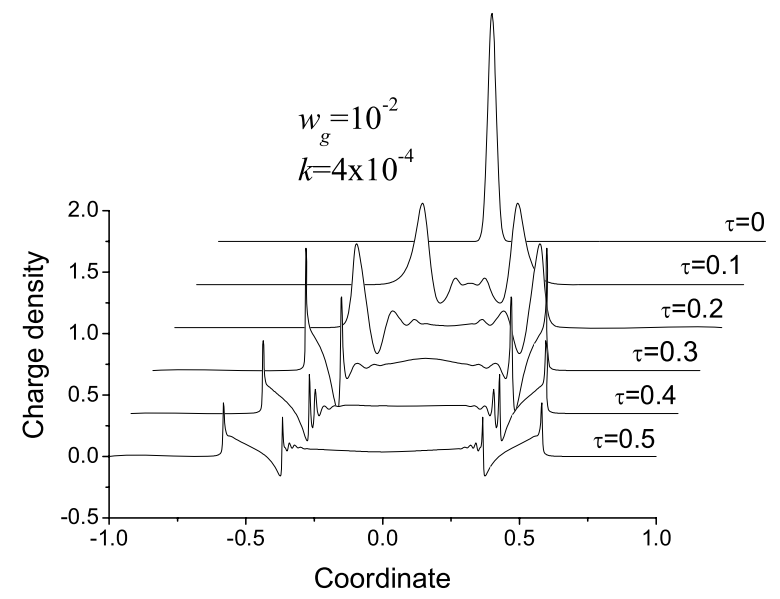

Figure 4: Evolution of spatial distribution of sheet charge in 2DEG channel with nonuniform donor distribution (effect of tsunami).

Due to the formation of the shock waves, the gate currents can be much faster than the input voltage signals. The propagating shock waves might induce rapidly varying charges in the gate sections. This can be used for generating of terahertz signals by relatively slow (with moderate values of $t_{0}$ ) electric signals.

\section{Acknowledgment}

One of the authors (V.R.) thanks T. Otsuji, A. Chaplik, and M. S. Shur for stimulating discussions. This work is supported by the Grant-in-Aid for Scientific Research (S) from the Japan Society for Promotion of Science, Japan.

\section{References}

[1] M. Dyakonov and M. Shur, "Detection, mixing, and frequency multiplication of terahertz radiation by two-dimensional electronic fluid," IEEE Trans. Electron. Devices, vol 43, pp. 1640-1645, 1996).

[2] C. A. J. Fletcher, "Computational Techniques for Fluid Dynamics",Springer, NY, 1988. 